Published in final edited form as:

J Mol Cell Cardiol. 2008 March ; 44(3): 571-581.

\title{
Cardiac Potassium Channel Dysfunction in Sudden Infant Death Syndrome
}

\author{
Troy E. Rhodes ${ }^{1}$, Robert A. Abraham ${ }^{1}$, Richard C. Welch ${ }^{1}$, Carlos G. Vanoye ${ }^{1}$, Lia Crotti 2,3 , \\ Marianne Arnestad ${ }^{4}$, Roberto Insolia ${ }^{2}$, Matteo Pedrazzini ${ }^{2}$, Chiara Ferrandi ${ }^{2}$, Ashild Vege ${ }^{4}$, \\ Torleiv Rognum ${ }^{4}$, Dan M. Roden ${ }^{1,6}$, Peter J. Schwartz $2,3,5$, and Alfred L. George Jr. 1,6 \\ 1Department of Medicine, Vanderbilt University, Nashville, TN, USA \\ 2Molecular Cardiology Laboratory, IRCCS Policlinico S. Matteo, Pavia, Italy \\ 3Department of Cardiology, University of Pavia and IRCCS Policlinico S. Matteo, Pavia, Italy \\ 4Institute of Forensic Medicine, University of Oslo, Oslo, Norway \\ 5Laboratory of Cardiovascular Genetics, IRCCS Istituto Auxologico, Milan, Italy \\ 6Department of Pharmacology, Vanderbilt University, Nashville, TN, USA
}

\section{Abstract}

Life-threatening arrhythmias have been suspected as one cause of the sudden infant death syndrome (SIDS), and this hypothesis is supported by the observation that mutations in arrhythmia susceptibility genes occur in 5-10\% of cases. However, the functional consequences of cardiac potassium channel gene mutations associated with SIDS and how these alleles might mechanistically predispose to sudden death are unknown. To address these questions, we studied four missense $K C N H 2$ (encoding HERG) variants, one compound $K C N H 2$ genotype, and a missense $K C N Q 1$ mutation all previously identified in Norwegian SIDS cases. Three of the six variants exhibited functional impairments while three were biophysically similar to wild-type channels $(\mathrm{KCNH} 2$ variants V279M, R885C, S1040G). When coexpressed with WT-HERG, R273Q and K897T/R954C generated currents resembling the rapid component of the cardiac delayed rectifier current $\left(\mathrm{I}_{\mathrm{Kr}}\right)$ but with significantly diminished amplitude. Action potential modeling demonstrated that this level of functional impairment was sufficient to evoke increased action potential duration and pausedependent early afterdepolarizations. By contrast, $K C N Q 1-\mathrm{I} 274 \mathrm{~V}$ causes a gain-of-function in $\mathrm{I}_{\mathrm{Ks}}$ characterized by increased current density, faster activation, and slower deactivation leading to accumulation of instantaneous current upon repeated stimulation. Action potential simulations using

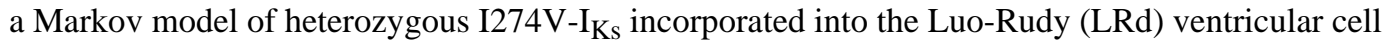
model demonstrated marked rate-dependent shortening of action potential duration predicting a short QT phenotype. Our results indicate that certain potassium channel mutations associated with SIDS confer overt functional defects consistent with either LQTS or SQTS, and further emphasize the role of congenital arrhythmia susceptibility in this syndrome.

Correspondence: Alfred L. George Jr., Division of Genetic Medicine, 529 Light Hall, 2215 Garland Avenue, Nashville, TN 37232-0275, Tel: 615-936-2660, Fax: 615-936-2661, Email: al.george@ vanderbilt.edu.

Publisher's Disclaimer: This is a PDF file of an unedited manuscript that has been accepted for publication. As a service to our customers we are providing this early version of the manuscript. The manuscript will undergo copyediting, typesetting, and review of the resulting proof before it is published in its final citable form. Please note that during the production process errors may be discovered which could affect the content, and all legal disclaimers that apply to the journal pertain.

Disclosures - The authors have no potential conflicts of interest. 


\section{Keywords}

sudden death; genetics; ion channels; long QT syndrome; short QT syndrome

\section{Introduction}

Life-threatening arrhythmias including the congenital long QT syndrome (LQTS) have been proposed to contribute to some cases of the sudden infant death syndrome (SIDS), the leading cause of death among infants 1 month to 1 year of age [1-3]. Supporting this theory are recent observations that mutations in genes responsible for LQTS are found in approximately 5-10\% of SIDS cases [4-6]. Congenital LQTS is an inherited disorder associated with mutations in genes predominantly encoding subunits of cardiac ion channels [7]. The disorder is caused by impaired ventricular repolarization leading to prolongation of the QT interval and an increased risk for life-threatening ventricular arrhythmias. Other mutations in some of the same genes $(K C N H 2, K C N Q 1)$ can enhance repolarization and cause shortening of the QT interval [810]. In the short QT syndrome (SQTS) there is also increased risk for cardiac arrhythmias and sudden death $[11,12]$.

The data linking cardiac potassium channel mutations with SIDS include anecdotal reports and population surveys. In 2001, Schwartz and colleagues reported discovery of a de novo $K C N Q 1$ mutation (P117L) in a SIDS victim [13]. The same mutation was also observed to segregate with autosomal dominant LQTS at reduced penetrance in an unrelated Italian family [13]. Another missense $K C N Q 1$ variant $(\mathrm{H} 105 \mathrm{~L})$ without functional consequences was discovered among 41 German SIDS cases [14]. Two novel KCNH2 mutations (K101E, P1157L) have also been reported in single SIDS probands [5,15], and in one case this finding led to discovery of undiagnosed LQTS in family members [15]. But, there have been no studies demonstrating that any of these mutant channels are dysfunctional.

Ackerman et al. reported a systematic survey of autopsied SIDS cases for mutations in the LQTS genes, an effort that originally identified two SCN5A mutations in 93 subjects [4]. The study later reported novel mutations in cardiac potassium channel genes $(K C N H 2-\mathrm{G} 294 \mathrm{~V}$, $K C N Q 1-\mathrm{T} 600 \mathrm{M})$ occurring in two SIDS victims [5]. Three other potassium channel variants were identified, but these alleles were also observed in ethnically-matched population controls, albeit rarely. Most recently, the largest series of cases screened for mutations in arrhythmiasusceptibility genes revealed novel missense $\mathrm{KCNH} 2$ and $\mathrm{KCNQ} 1$ mutations in Norwegian SIDS victims [6]. Importantly, the electrophysiological consequences of these novel SIDSassociated potassium channel mutations were previously unknown.

We report here the functional characterization of novel $K C N H 2$ and $K C N Q 1$ mutations identified in the Norwegian SIDS study. Our findings demonstrated a diversity of functional phenotypes that revealed plausible explanations for sudden death in this clinical setting.

\section{Materials and Methods}

\subsection{Mutagenesis and heterologous expression}

Four single $K C N H 2$ variants (V279M, R273Q, R885C, S1040G), one compound genotype (K897T/R954C) and a KCNQ1 mutation (I274V) were constructed in HERG or KCNQ1 cDNA vectors, respectively, using recombinant PCR mutagenesis (primer sequences available upon request). The final constructs were assembled in bicistronic mammalian expression plasmids (pIRES2-EGFP or pIRES2-DsRed, BD Biosciences-Clontech, Palo Alto, CA) to enable monitoring of transfection success by co-expression of fluorescent proteins. All constructs were sequenced to verify the mutation and to exclude polymerase errors. Recombinant human 
KCNE1 cDNA was subcloned into pIRES2-DsRed for use in $\mathrm{I}_{\mathrm{Ks}}$ expression experiments. In co-expression experiments, the WT channel (either KCNQ1 or HERG) was coupled to DsRed while mutants were expressed from vectors encoding EGFP.

Chinese hamster ovary cells (CHO-K1, ATCC) cells were grown as previously described $[16,17]$. For HERG experiments, cells were transiently co-transfected with $3 \mu \mathrm{g}$ of both WT and mutant plasmid DNA using Fugene-6 (Roche Diagnostics, Indianapolis, IN). For KCNQ1 experiments, cells were transiently transfected with $1 \mu \mathrm{g}$ KCNQ1 plasmid DNA, whereas to study $\mathrm{I}_{\mathrm{Ks}}$, cells were co-transfected with $1 \mu \mathrm{g} \mathrm{KCNQ} 1$ and $1 \mu \mathrm{g}$ KCNE1 plasmids. In some experiments, we performed transient transfection of WT or mutant KCNQ1 in a stable $\mathrm{I}_{\mathrm{Ks}}$ cell line [17]. For these experiments, we initially determined by plasmid DNA titration that $1 \mathrm{ng}$ of WT-KCNQ1 was sufficient to boost current amplitude in stable $\mathrm{I}_{\mathrm{Ks}}$ cells by 2-3 fold and thus used this amount in transfections to achieve approximately equal levels of stably and transiently expressed KCNQ1 alleles. Following transfection (48-72 hours), fluorescent cells were selected by epifluorescence microscopy (green for single transfections, yellow for cotransfections) for use in whole-cell patch clamp recording experiments. Non-transfected cells grown under these conditions did not exhibit measurable endogenous potassium currents.

\subsection{Electrophysiology}

Whole-cell currents were measured in the whole-cell configuration of the patch clamp technique [18] using an Axopatch 200B amplifier (Molecular Devices, Corp., Sunnyvale, CA, USA) as previously described [16,17]. Patch pipettes were pulled from thick-wall borosilicate glass (World Precision Instruments, Inc., Sarasota, FL, USA) with a multistage P-97 FlamingBrown micropipette puller (Sutter Instruments Co., San Rafael, CA, USA) and fire-polished to a pipette resistance of 2-4 MS. A 1-2\% agar-bridge composed of bath solution was utilized as a reference electrode. For HERG recording, the bath solution consisted of (in $\mathrm{mM}$ ): $\mathrm{NaCl}$ $145, \mathrm{KCl} 4, \mathrm{MgCl}_{2} 1, \mathrm{CaCl}_{2} 1.8$, glucose 10 , HEPES 10, adjusted to $\mathrm{pH} 7.35$ with $\mathrm{NaOH}, \sim 275$ $\mathrm{mosm} / \mathrm{kg}$. The pipette solution consisted of (in $\mathrm{mM}$ ): $\mathrm{KCl} 110$, ATP (dipotassium salt) 5 , $\mathrm{MgCl}_{2}$ 2, EDTA (ethylenediaminetetraacetic acid) 10, HEPES 10, adjusted to $\mathrm{pH} 7.2$ with $\mathrm{KOH}, \sim 265 \mathrm{mosmol} / \mathrm{kg}$. For KCNQ1 and $\mathrm{I}_{\mathrm{Ks}}$ recording, the bath solution consisted of (in $\mathrm{mM}$ ): $\mathrm{NaCl} 132, \mathrm{KCl} 4.8, \mathrm{MgCl}_{2} 1.2, \mathrm{CaCl}_{2} 2$, glucose 5, HEPES 10, $\mathrm{pH} 7.4, \sim 275 \mathrm{mosm} / \mathrm{kg}$. The pipette solution consisted of (in $\mathrm{mM}$ ): K-aspartate 110, ATP (dipotassium salt) $5, \mathrm{MgCl}_{2} 1$, EGTA 11, HEPES 10, $\mathrm{MgCl}_{2} 1, \mathrm{pH} 7.3, \sim 265 \mathrm{mosm} / \mathrm{kg}$. Pipette solutions were diluted with distilled water (to $\sim 250 \mathrm{mosm} / \mathrm{kg}$ ) to prevent activation of swelling-activated currents. Chemicals were purchased from SIGMA (St. Louis, MO, USA).

For HERG experiments, the holding potential was $-80 \mathrm{mV}$ and whole-cell currents were measured from -80 to $+70 \mathrm{mV}$ (in $10 \mathrm{mV}$ steps) $1990 \mathrm{~ms}$ (activation) and $2200 \mathrm{~ms}$ (tail currents) after the start of the voltage pulse. For KCNQ1 and $\mathrm{I}_{\mathrm{Ks}}$ experiments, the holding potential was $-80 \mathrm{mV}$ and whole-cell currents were measured from -80 to $+60 \mathrm{mV}$ (in $10 \mathrm{mV}$ steps) $1990 \mathrm{~ms}$ after the start of the voltage pulse. Whole-cell current traces were filtered at 5 $\mathrm{kHz}$ and acquired at $2 \mathrm{kHz}$. The access resistance and apparent membrane capacitance were estimated as described by Lindau and Neher [19]. Pulse generation, data collection and analyses were done with Clampex 8.1 (Molecular Devices, Corp.). Specific voltage pulse protocols are described in the figures and legends. The apparent voltage-dependence of activation for $\mathrm{I}_{\mathrm{Ks}}$ was obtained by fitting data with the Boltzmann function: $\mathrm{I}=1 /\left(1+\exp \left(\mathrm{V}-\mathrm{V}_{1 / 2}\right) / \mathrm{k}\right)$. To quantify the time course of deactivation, tail currents were fit with a single exponential function, $\mathrm{f}(\mathrm{t})=\mathrm{A}_{1} \cdot \exp \left(-\mathrm{t} / \tau_{1}\right)+C$. Statistical comparisons were made using Student's t-test and significance was assumed for $P<0.05$. 


\subsection{Computational Modeling}

Markov models of human WT and mutant $\mathrm{I}_{\mathrm{Ks}}$ and $\mathrm{I}_{\mathrm{Kr}}$ were applied to the LRd simulation of ventricular action potentials. In studies of $\mathrm{I}_{\mathrm{Ks}}$, only $\mathrm{I}_{\mathrm{Ks}}$ was modified in the simulation; similarly, in studies of $\mathrm{I}_{\mathrm{Kr}}$, only $\mathrm{I}_{\mathrm{Kr}}$ was modified. Simulations were performed at $310^{\circ} \mathrm{K}\left(37^{\circ}\right.$ C), with a $\mathrm{Q}_{10}$ of 3 applied to each $\mathrm{I}_{\mathrm{Ks}}$ and $\mathrm{I}_{\mathrm{Kr}}$ Markov transition rate to account for the experimental data being recorded at $296^{\circ} \mathrm{K}\left(23^{\circ} \mathrm{C}\right)$.

Markov models of wild-type and mutant $\mathrm{I}_{\mathrm{Kr}}$ were generated using the 5-state formulation described by Clancy and Rudy (illustrated as an inset in Fig. 2A) [20]. The WT- $\mathrm{I}_{\mathrm{Kr}}$ model was generated by optimizing simultaneously all 6 Markov transition rates to minimize the leastsquared difference between modeled and experimental current density curves (see supplemental Fig. S1). To generate R273Q- $\mathrm{I}_{\mathrm{Kr}}$, each Markov transition rate was optimized independently. The single transition rate optimization that gave the best match with experimental data (assessed by $\chi^{2}$ ) was chosen. An amplitude normalization factor was chosen for WT-I $\mathrm{I}_{\mathrm{Kr}}$ such that, when applied to LRd model, an APD of $180 \mathrm{~ms}$ was achieved at a paced cycle length of $1000 \mathrm{~ms}$. The same amplitude normalization factor was then applied to the $\mathrm{R} 273 \mathrm{Q}-\mathrm{I}_{\mathrm{Kr}}$ model. The model parameters and transition rate equations are provided in supplemental Table S1.

Markov models of wild-type $\mathrm{I}_{\mathrm{Ks}}\left(\mathrm{WT}-\mathrm{I}_{\mathrm{Ks}}\right.$ ) and $\mathrm{I} 274 \mathrm{~V}-\mathrm{I}_{\mathrm{Ks}}$ were generated using the 17 -state formulation described by Silva and Rudy [21] (see supplemental Fig. S2). The model parameters and transition rate equations are provided in supplemental Table S2. To generate the WT-I $\mathrm{I}_{\mathrm{Ks}}$ model, all 17 Markov transition rates were optimized to minimize the least-squared difference between modeled and experimental current density curves. To model heterozygous $\mathrm{I} 274 \mathrm{~V}-\mathrm{I}_{\mathrm{Ks}}$, we started with the WT-I $\mathrm{K}_{\mathrm{Ks}}$ model and then each Markov transition rate was optimized independently. The single transition rate optimization that gave the best match with experimental data (assessed by $\chi^{2}$ ) was chosen. Because a main feature of repetitive I274V$\mathrm{I}_{\mathrm{Ks}}$ channel stimulation was current accumulation, multiple traces within a single voltage protocol were compared within each optimization iteration. Capacitive transients were not allowed to contribute to the $\chi^{2}$ comparison. The set of adjustments to the transition rates and conductance (see supplemental Table S2) that minimized the $\chi^{2}$ difference between experimental and simulated data (see supplemental Fig. S3) was used to form the model for all subsequent WT- $\mathrm{I}_{\mathrm{Ks}}$ simulations. An amplitude normalization factor was chosen for WT$\mathrm{I}_{\mathrm{Ks}}$ such that, when applied to the Luo-Rudy (LRd) model, an action potential duration (APD) of $200 \mathrm{~ms}$ was achieved at a paced cycle length of $1000 \mathrm{~ms}$. The same amplitude normalization factor was then applied to the $\mathrm{I} 274 \mathrm{~V}-\mathrm{I}_{\mathrm{Ks}}$ model.

Steady-state action potential simulations were recorded with pacing cycle lengths ranging from 300 to $10000 \mathrm{~ms}$. The effects of a preceding pause on action potential duration was examined for WT and mutant $\mathrm{I}_{\mathrm{Kr}}$. For these simulations, a $1000 \mathrm{~ms}$ pacing cycle length protocol was conducted to steady state. The final pacing stimulus was preceded by a pause of specified duration, and the difference between the duration of that action potential and that of the preceding action potential (without an antecedent pause) was recorded. All simulations were implemented using Matlab v7.3 (The Mathworks, Inc., Natick, MA) with $\mathrm{C}^{++}$code generously provided by Jonathan Silva and Yoram Rudy and run on a Macintosh G5-quad.

\section{Results}

We examined the biophysical properties of four single missense $\mathrm{KCNH} 2$ variants, one compound $\mathrm{KCNH} 2$ genotype, and a missense $K C N Q 1$ mutation using whole-cell patch clamp recording of recombinant potassium channels heterologously expressed in cultured mammalian cells. All mutations were originally identified in a large, retrospective series of 201 Norwegian SIDS cases that were screened for genetic variants in the major LQTS genes 
[6]. Three $K C N H 2$ alleles (V279M, R885C, S1040G) exhibited biophysical properties indistinguishable from WT-HERG and we infer that these are probably benign variants. However, a range of functional defects were observed among the other mutations.

\subsection{Partial loss-of-function $\mathrm{KCNH} 2$ variants}

Two KCNH2 mutations, R273Q and R954C, alter highly conserved amino acid residues in the $\mathrm{N}$-terminus or $\mathrm{C}$-terminus of the HERG potassium channel, respectively. The proband in whom R954C was discovered also carried the common polymorphism K897T on the mutant allele giving rise to the compound genotype, K897T/R954C. Since each proband also carried a WT allele, we functionally characterized each mutant by co-expression with WT-HERG. To demonstrate expression of both constructs in the same cells, bicistronic vectors coupling the variant HERG allele to EGFP and WT-HERG to DsRed enabled the identification of coexpressing cells by yellow fluorescence (combined EGFP and DsRed).

Figure 1A illustrates representative whole-cell currents in cells co-expressing WT-HERG with either HERG-R273Q or HERG-K897T/R954C. When co-expressed with WT-HERG, both mutants generated currents resembling the rapid component of the cardiac delayed rectifier current $\left(\mathrm{I}_{\mathrm{Kr}}\right.$ ). However, the amplitude of the activating (Fig. 1B) and peak tail currents (Fig. 1C) were significantly smaller at positive potentials in cells expressing the mutants as compared with WT-HERG alone. Tail current density was reduced $\sim 35 \%$ and $\sim 50 \%$ for cells expressing HERG-R273Q and HERG-K897T/R954C, respectively. Our results indicated that these two SIDS-associated $\mathrm{KCNH} 2$ variants confer partial loss-of-function.

To determine if this degree of HERG functional impairment was sufficient to cause abnormal myocardial repolarization, we employed computational modeling of cardiac action potentials using the LRd model. Specifically, we modeled the level of HERG dysfunction observed for R273Q which was less severe. We first optimized Markov models for both WT and the R273Q using data derived from Fig. 1 (see supplemental Fig. S1), and then performed action potential simulations using different cycle lengths. Figure $2 \mathrm{~A}$ illustrates that the mutant exhibits increased APD across a wide range of simulated cycle lengths. Because impaired HERG function is often associated with pause-dependent ventricular arrhythmias [22], we simulated the impact of an inter-pulse pause of varying duration. In Fig. 2B, we illustrate that progressive lengthening of the pause evokes lengthening of the APD and the spontaneous occurrence of an early afterdepolarization (EAD). We never observed an EAD in action potential simulations using the $\mathrm{WT} \mathrm{I}_{\mathrm{Kr}}$ model. These findings support the notion that reduced repolarization reserve conferring increased risk of arrhythmogenic electrical activity can occur with certain

$K C N H 2$ mutations associated with SIDS.

\subsection{KCNQ1-I274V is a gain-of-function mutation}

The mutation KCNQ1-I274V, which alters a highly conserved amino acid residue in the S5 segment of the KCNQ1 channel, confers a unique biophysical phenotype. Figure $3 \mathrm{~A}$ illustrates representative whole-cell currents evoked by a series of depolarizing test potentials in cells expressing either WT-KCNQ1 or KCNQ1-I274V. In the absence of KCNE1, KCNQ1-I274V generated a voltage dependent, rapidly activating, and slowly deactivating outward potassium current with current density (Fig. 3B) and biophysical properties similar to WT-KCNQ1.

However, when expressed with KCNE1 (Fig. 3C), KCNQ1-I274V generated typical slowly activating outward currents resembling $\mathrm{I}_{\mathrm{Ks}}$, but with significantly greater peak current density than $\mathrm{WT}^{-\mathrm{I}_{\mathrm{Ks}}}$ at test potentials positive to $-30 \mathrm{mV}$ (Fig. 3D). The increase in peak current density observed for $\mathrm{I} 274 \mathrm{~V}-\mathrm{I}_{\mathrm{Ks}}$ could not be explained by a simple shift in the voltage dependence of activation (apparent $\mathrm{V}_{1 / 2}$ values from Boltzmann fits of isochronal activation

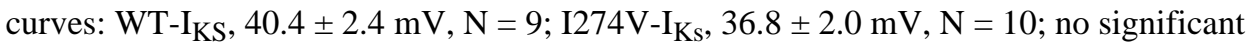
difference). 


\subsection{Altered activation and deactivation kinetics of $1274 \mathrm{~V}-\mathrm{I}_{\mathrm{Ks}}$}

In whole cell recordings, $\mathrm{I} 274 \mathrm{~V}-\mathrm{I}_{\mathrm{Ks}}$ appeared to have more rapid activation compared to WT$\mathrm{I}_{\mathrm{Ks}}$ (Fig. 3C). These differences in activation are further illustrated in Fig. 4A with representative $\mathrm{WT}-\mathrm{I}_{\mathrm{Ks}}$ and $\mathrm{I} 274 \mathrm{~V}-\mathrm{I}_{\mathrm{Ks}}$ traces that were normalized to their respective peak current levels. We quantified activation kinetics by measuring the time to half-maximal current at each voltage and observed significantly faster activation for $\mathrm{I} 274 \mathrm{~V}-\mathrm{I}_{\mathrm{Ks}}$ at positive test potentials between 20 and $50 \mathrm{mV}$ (Fig. 4B).

The time course of deactivation was also slower for $\mathrm{I} 274 \mathrm{~V}-\mathrm{I}_{\mathrm{Ks}}$ compared to WT-I $\mathrm{Ks}$ (Fig. 3D). To further investigate the kinetics and voltage dependence of deactivation for $\mathrm{I} 274 \mathrm{~V}-\mathrm{I}_{\mathrm{Ks}}$, cells were depolarized to $40 \mathrm{mV}$ followed by repolarization to a range of potentials from -40 to $-100 \mathrm{mV}$. Figure 5A illustrates tail current deactivation for $\mathrm{WT}-\mathrm{I}_{\mathrm{Ks}}$ and $\mathrm{I} 274 \mathrm{~V}-\mathrm{I}_{\mathrm{Ks}}$ in representative traces normalized to the peak current measured at each voltage. Differences in the deactivation time course between $\mathrm{WT}-\mathrm{I}_{\mathrm{Ks}}$ and $\mathrm{I} 274 \mathrm{~V}-\mathrm{I}_{\mathrm{Ks}}$ are further illustrated in Fig. 5B. Tail currents were fit by a single exponential equation to obtain deactivation time constants $\left(\tau_{\text {deact }}\right)$. The time course of deactivation was significantly slower for $\mathrm{I} 274 \mathrm{~V}-\mathrm{I}_{\mathrm{Ks}}$, as evidenced by the larger time constants measured at -80 to $-100 \mathrm{mV}$ (Fig. 5C). Deactivation time constants could not be measured at $-70 \mathrm{mV}$ since this voltage is close to the equilibrium potential for potassium under our experimental conditions, and no measurable deactivation was observed for $\mathrm{I} 274 \mathrm{~V}-\mathrm{I}_{\mathrm{Ks}}$ at $-60 \mathrm{mV}$.

\subsection{Gain-of-function effect of KCNQ1-I274V is dominant}

Because KCNQ1-I274V was heterozygous in the SIDS proband [6], we tested whether this mutation conferred a dominant gain-of-function effect by co-expressing either WT-KCNQ1 or KCNQ1-I274V channels together with KCNE1 in the same cells. To avoid cell-to-cell variability associated with the transient transfection of three independent plasmids, we transiently expressed KCNQ1-I274V (engineered in an IRES2-EGFP vector) in a cell line stably expressing WT-KCNQ1 and KCNE1 ( $\mathrm{I}_{\mathrm{Ks}}$ cells). This strategy permitted reliable examination of cells expressing all three cDNAs. We initially optimized transfection conditions to determine the amount of WT-KCNQ1 plasmid DNA necessary to boost $\mathrm{I}_{\mathrm{Ks}}$ current density by just $2-3$ fold to avoid a large allelic imbalance. Figure 6 illustrates a comparison of wholecell currents from $\mathrm{I}_{\mathrm{Ks}}$ cells transiently transfected with either WT or I274V. Expression of the mutant channel causes a significantly greater current density consistent with a dominant gainof-function phenotype.

The abnormal activation and deactivation kinetics observed for I274V-I $\mathrm{I}_{\mathrm{Ks}}$ (Fig. 4 and Fig. 5) suggested that the mutation may modulate rate-dependent responses of the current. To test this hypothesis, we examined the behavior of WT and mutant channels in response to a series of 50 depolarizing pulses $\left(2 \mathrm{~s}, 60 \mathrm{mV}\right.$ ) using the $\mathrm{I}_{\mathrm{Ks}}$ cell expression strategy described above (Fig. $7 \mathrm{~A})$. With each successive pulse, $\mathrm{I}_{\mathrm{Ks}}$ cells transfected with WT-KCNQ1 generated typical slowly activating outward currents with only a slight potentiation of current amplitude. By contrast in $\mathrm{I}_{\mathrm{Ks}}$ cells transfected with $\mathrm{I} 274 \mathrm{~V}$, the typical slowly activating outward current was observed only with the first pulse, and with subsequent pulses, a rapidly activating current was observed that progressively increased in amplitude. Deactivation during the post-pulse recovery step to $-30 \mathrm{mV}$ was also noticeably slower in cells expressing $1274 \mathrm{~V}$.

To further quantify the abnormal rate dependent behavior exhibited by cells expressing I274V, currents were measured at $150 \mathrm{~ms}$ ("instantaneous") and $1990 \mathrm{~ms}$ ("late") after the start of the depolarizing pulse (see arrows on voltage protocol, Fig. 7A). The accumulation of instantaneous and late current was much larger for cells expressing I274V (Figs. 7B), consistent with the slower deactivation kinetics observed for the mutant. These studies support the idea 
that impaired deactivation of $1274 \mathrm{~V}$ can contribute to accumulation of the instantaneous current with repeated stimulation.

\subsection{Action potential modeling of I274V}

The electrophysiological data indicated that in the presence of KCNE1, KCNQ1-I274V causes a gain-of-function in $\mathrm{I}_{\mathrm{Ks}}$ characterized by increased current density, faster activation, slower deactivation, and accumulation of instantaneous current during repeated stimulation.

Therefore, we hypothesized that I274V may promote a short QT syndrome phenotype. To test this hypothesis, computational modeling of ventricular action potentials was performed using a modified LRd model [21] in which a Markov modeling approach was applied to simulate WT- $\mathrm{I}_{\mathrm{Ks}}$ and $\mathrm{I} 274 \mathrm{~V}-\mathrm{I}_{\mathrm{Ks}}$ from data collected from the $\mathrm{I}_{\mathrm{Ks}}$ cell experiments (Fig. 6 and Fig. 7). Implementation of WT-I $\mathrm{K}_{\mathrm{Ks}}$ alone into the LRd model evoked simulated action potentials similar to those published previously [21]. To model I274V-I $\mathrm{I}_{\mathrm{Ks}}$, we systematically modified each individual rate constant and evaluated the resulting simulated current profiles to determine the best fit to experimental data. Changing a single Markov transition rate was sufficient to best approximate steady-state I274V-I $\mathrm{I}_{\mathrm{Ks}}$ behavior (see supplemental Table S2). Comparison of LRd simulations between $\mathrm{WT}-\mathrm{I}_{\mathrm{Ks}}$ and heterozygous $\mathrm{I} 274 \mathrm{~V}-\mathrm{I}_{\mathrm{Ks}}$ revealed action potential shortening at all cycle lengths with little change in action potential morphology (Fig. 8). These simulations support our prediction that $K C N Q 1-\mathrm{I} 274 \mathrm{~V}$ will cause a short QT phenotype, a plausible explanation for sudden death in an infant carrying this mutation.

\section{Discussion}

Sudden unexplained death of an infant is a tragic event. In the absence of an obvious cause of death including intentional injury as demonstrated by a negative autopsy and a thorough death scene investigation, a diagnosis of sudden infant death syndrome (SIDS) can be considered [23]. Although, the diagnosis of SIDS is one of exclusion, ongoing efforts to define preventable causes have great importance for understanding the pathogenesis of this syndrome and ultimately for reducing infant mortality. But despite intense research efforts to uncover its etiologies, SIDS remains a leading cause of death during the first year of life in industrialized nations [24-27]. In this study, our findings demonstrate that mutations in the cardiac potassium channel genes $K C N Q 1$ and $K C N H 2$ discovered in SIDS cases can exhibit functional defects consistent with either LQTS or SQTS. These observations strengthen the notion that congenital arrhythmia susceptibility may contribute to the pathogenesis of sudden infant death and that these disorders may present clinically as SIDS.

\subsection{Cardiac potassium channel dysfunction in SIDS}

The consequences of $K C N Q 1$ and $K C N H 2$ mutations associated with SIDS were not previously known [5,13-15], but we hypothesized that significant functional impairments were likely in some cases. However, it was difficult to predict a priori which variants would cause potassium channel dysfunction and promote arrhythmia susceptibility. In our report on the prevalence of LQTS gene mutations in Norwegian SIDS subjects [6], the three $K C N H 2$ variants (V279M, R885C, S1040G) that did not exhibit overt functional effects were not considered in calculating the percentage of SIDS cases associated with a pathological mutation but this had been based on preliminary work. Here we present the complete functional characterization of several mutants including those that exhibit abnormal functional properties that plausibly contributed to an increased risk of sudden infant death.

\subsection{Loss-of-function $\mathrm{KCNH} 2$ variants in SIDS}

Our studies demonstrated that two $\mathrm{KCNH} 2$ variant genotypes conferred partial loss-of-function consistent with LQTS and potentially contributed to unexplained death in the SIDS victims who carried these alleles. In comparison to other mutations associated with LQTS, the 
functional impairments observed for $K C N H 2-\mathrm{R} 273 \mathrm{Q}$ and the compound variant $\mathrm{KCNH} 2$ K897T/R954C are considerably less severe. We speculate that the degree of functional loss exhibited by these SIDS-associated genotypes may require additional genetic or environmental factors to promote arrhythmogenesis. This notion was first demonstrated in the context of SCN5A variants associated with SIDS by Plant and colleagues for African-American carriers of SCN5A-S1103Y [28] and later emphasized by our study of several SCN5A mutations identified in the Norwegian SIDS cohort [29]. In those reports, exposure of seemingly benign sodium channel variants to intracellular acidosis unmasked a latent functional defect compatible with increased arrhythmia susceptibility. Other genetic factors may also contribute to sudden death in SIDS victims carrying $\mathrm{KCNH} 2$ variants that exhibit mild functional impairments [16].

\subsection{Novel gain-of-function KCNQ1 mutation in SIDS}

The most well studied congenital arrhythmia susceptibility syndrome is LQTS and a subset of SIDS cases may be caused by similar pathophysiological mechanisms. However, other inherited arrhythmia syndromes may also contribute to sudden death during infancy. Here, we demonstrated that $K C N Q 1-\mathrm{I} 274 \mathrm{~V}$ is a novel gain-of-function mutation that is predicted to cause shortening of the ventricular action potential and the SQTS, another recognized predisposition for re-entrant arrhythmias and associated with an increased risk of sudden cardiac death. Indeed, aborted cardiac arrest in an 8-month old infant was reported in a carrier of $\mathrm{KCNH}_{2}$ N588K in one of the first described SQTS families $[8,12]$. However, sudden infant death has not been previously associated with gain-of-function $K C N Q 1$ mutations in either SQTS (V141M, V307L) [9,30] or atrial fibrillation (S140G) [31], but the proband first identified with $K C N Q 1-\mathrm{V} 141 \mathrm{M}$ presented in utero with an irregular bradyarrhythmia, which was recognized as atrial fibrillation, and SQTS manifested in the postnatal period [30].Unfortunately, we cannot confirm SQTS in the Norwegian SIDS victim in whom KCNQ1-I274V was discovered or in first degree family members because all Norwegian SIDS victims were anonymous. But a fatal ventricular arrhythmia promoted by an abbreviated action potential seems a likely explanation for sudden death in this infant.

The structural basis for a gain-of-function associated with $K C N Q 1$-I274V was not specifically explored by our study. The mutation affects a conserved residue in the S5 segment which contributes to the pore domain directly, but is also connected to the voltage-sensor domain by the S4-S5 linker. It is possible that gain-of-function is the result of increased single channel conductance, increased numbers of channels in the plasma membrane, or an increase in channel open probability. Based on the abnormal deactivation kinetics observed for I274V, we speculate that an increase in open probability is quite likely. We also observed that the gainof-function was dependent upon the presence of the accessory subunit KCNE1. A similar KCNE1-dependence was reported for the SQTS mutant KCNQ1-V141M [30]. A molecular explanation for how the I274V mutation in the S5 segment alters KCNQ1 gating modulation by KCNE1 is beyond the scope of our current study, but future studies aimed at understanding this phenomenon may provide information about fundamental aspects of this subunit interaction.

\subsection{Functional impact of altered $I_{\mathrm{Ks}}$ deactivation}

The kinetics of $\mathrm{I}_{\mathrm{Ks}}$ deactivation may determine the rate of $\mathrm{I}_{\mathrm{Ks}}$ accumulation during faster heart rates accounting for action potential duration adaptation in some species [32]. However, a recent computational study demonstrated that negligible open-state $\mathrm{I}_{\mathrm{Ks}}$ accumulation occurs at rapid heart rates in human heart, but instead, the channels accumulate in pre-open closed states as an available reserve [21]. Our findings demonstrate that impaired deactivation of I274V-I $\mathrm{Ks}_{\mathrm{Ks}}$ leads to rate-dependent accumulation of open channels. At rapid heart rates, such as those typical of infancy, impaired deactivation may lead to $\mathrm{I}_{\mathrm{Ks}}$ accumulation and pronounced 
shortening of the action potential duration. This effect may exaggerate the transmural dispersion of repolarization that normally exists between epicardial and endocardial layers and thus promote arrhythmia susceptibility [33].

\subsection{Study limitations}

Our study utilizes heterologous expression of recombinant human ion channels. Although this approach is widely employed to assess functional consequences of ion channel mutations, we recognize that the in vivo effect of mutant potassium channels may be further influenced by factors in the native human cardiac myocyte environment that can't be replicated in vitro. Also, our computational studies used an established action potential model based upon guinea pig cardiac electrophysiology and direct relevance to human heart is not absolutely certain. However, we view these experiments collectively as supporting the hypothesis that certain cardiac potassium channel mutations identified in SIDS victims can confer increased arrhythmia susceptibility and this advances the concept that undiagnosed congenital arrhythmia syndromes can present as SIDS. Lastly, our work did not address the more complex issue of arrhythmia triggering mechanisms that may be important for understanding why some LQTS and SQTS mutation carriers do not survive infancy.

In conclusion, we demonstrated that there is a spectrum of cardiac potassium channel dysfunction associated with $K C N H 2$ and $K C N Q 1$ variants associated with SIDS that predict susceptibility to ventricular arrhythmias on the basis of either impaired or enhanced myocardial repolarization. These findings further establish that certain $\mathrm{KCNH} 2$ and $\mathrm{KCNQ} 1$ mutations are risk factors for SIDS, and emphasize SQTS as a potential cause of sudden infant death.

\section{Supplementary Material}

Refer to Web version on PubMed Central for supplementary material.

Acknowledgments

We thank Drs. Jonathan Silva and Yoram Rudy for helpful discussions regarding action potential modeling. This work was supported in part by NIH grant HL083374 (A.L.G.) and T.E.R. was supported by an institutional postdoctoral training grant (T32-GM007569).

\section{References}

1. Schwartz PJ. Cardiac sympathetic innervation and the sudden infant death syndrome. A possible pathogenetic link. Am J Med 1976;60:167-172. [PubMed: 175654]

2. Southall DP, Arrowsmith WA, Oakley JR, McEnery G, Anderson RH, Shinebourne EA. Prolonged QT interval and cardiac arrhythmias in two neonates: sudden infant death syndrome in one case. Arch Dis Child 1979;54:776-779. [PubMed: 507901]

3. Maron BJ, Clark CE, Goldstein RE, Epstein SE. Potential role of QT interval prolongation in sudden infant death syndrome. Circulation 1976;54:423-430. [PubMed: 947572]

4. Ackerman MJ, Siu BL, Sturner WQ, Tester DJ, Valdivia CR, Makielski JC, et al. Postmortem molecular analysis of SCN5A defects in sudden infant death syndrome. JAMA 2001;286:2264-2269. [PubMed: 11710892]

5. Tester DJ, Ackerman MJ. Sudden infant death syndrome: how significant are the cardiac channelopathies? Cardiovasc Res 2005;67:388-396. [PubMed: 15913580]

6. Arnestad M, Crotti L, Rognum TO, Insolia R, Pedrazzini M, Ferrandi C, et al. Prevalence of Long-QT Syndrome Gene Variants in Sudden Infant Death Syndrome. Circulation 2007;115:361-367. [PubMed: 17210839]

7. Schwartz, PJ.; Priori, SG.; Long, QT. syndrome: genotype-phenotype correlations. In: Zipes, DP.; Jalife, J., editors. Cardiac Electrophysiology From Cell to Bedside. 4th. Philadelphia, W.B.: Saunders Co.; 2004. p. 651-659. 
8. Brugada R, Hong K, Dumaine R, Cordeiro J, Gaita F, Borggrefe M, et al. Sudden death associated with short-QT syndrome linked to mutations in HERG. Circulation 2004;109:30-35. [PubMed: 14676148]

9. Bellocq C, van Ginneken AC, Bezzina CR, Alders M, Escande D, Mannens MM, et al. Mutation in the KCNQ1 gene leading to the short QT-interval syndrome. Circulation 2004;109:2394-2397. [PubMed: 15159330]

10. Priori SG, Pandit SV, Rivolta I, Berenfeld O, Ronchetti E, Dhamoon A, et al. A Novel Form of Short QT Syndrome (SQT3) Is Caused by a Mutation in the KCNJ2 Gene. Circ Res 2005;96:800-807. [PubMed: 15761194]

11. Gussak I, Brugada P, Brugada J, Wright RS, Kopecky SL, Chaitman BR, et al. Idiopathic short QT interval: a new clinical syndrome? Cardiology 2000;94:99-102. [PubMed: 11173780]

12. Gaita F, Giustetto C, Bianchi F, Wolpert C, Schimpf R, Riccardi R, et al. Short QT Syndrome: a familial cause of sudden death. Circulation 2003;108:965-970. [PubMed: 12925462]

13. Schwartz PJ, Priori SG, Bloise R, Napolitano C, Ronchetti E, Piccinini A, et al. Molecular diagnosis in a child with sudden infant death syndrome. Lancet 2001;358:1342-1343. [PubMed: 11684219]

14. Wedekind H, Bajanowski T, Friederich P, Breithardt G, Wulfing T, Siebrands C, et al. Sudden infant death syndrome and long QT syndrome: an epidemiological and genetic study. Int J Legal Med 2006;120:129-137. [PubMed: 16012827]

15. Christiansen M, Tonder N, Larsen LA, Andersen PS, Simonsen H, Oyen N, et al. Mutations in the HERG K ${ }^{+}$-ion channel: A novel link between long QT syndrome and sudden infant death syndrome. Am J Cardiol 2005;95:433-434. [PubMed: 15670565]

16. Crotti L, Lundquist AL, Insolia R, Pedrazzini M, Ferrandi C, De Ferrari GM, et al. KCNH2-K897T is a genetic modifier of latent congenital long-QT syndrome. Circulation 2005;112:1251-1258. [PubMed: 16116052]

17. Lundquist AL, Manderfield LJ, Vanoye CG, Rogers CS, Donahue BS, Chang PA, et al. Expression of multiple KCNE genes in human heart may enable variable modulation of $\mathrm{I}_{\mathrm{Ks}}$. J Mol Cell Cardiol 2005;38:277-287. [PubMed: 15698834]

18. Hamill OP, Marty A, Neher E, Sakmann B, Sigworth FJ. Improved patch-clamp techniques for highresolution current recording from cells and cell-free membrane patches. Pflügers Arch 1981;391:85100 .

19. Lindau M, Neher E. Patch-clamp techniques for time-resolved capacitance measurements in single cells. Pflugers Arch 1988;411:137-146. [PubMed: 3357753]

20. Clancy CE, Rudy Y. Cellular consequences of HERG mutations in the long QT syndrome: precursors to sudden cardiac death. Cardiovasc Res 2001;50:301-313. [PubMed: 11334834]

21. Silva J, Rudy Y. Subunit interaction determines $I_{K s}$ participation in cardiac repolarization and repolarization reserve. Circulation 2005;112:1384-1391. [PubMed: 16129795]

22. Tan HL, Bardai A, Shimizu W, Moss AJ, Schulze-Bahr E, Noda T, et al. Genotype-specific onset of arrhythmias in congenital long-QT syndrome: possible therapy implications. Circulation 2006;114:2096-2103. [PubMed: 17088455]

23. Krous HF, Beckwith JB, Byard RW, Rognum TO, Bajanowski T, Corey T, et al. Sudden infant death syndrome and unclassified sudden infant deaths: a definitional and diagnostic approach. Pediatrics 2004;114:234-238. [PubMed: 15231934]

24. Daley KC. Update on sudden infant death syndrome. Curr Opin Pediatr 2004;16:227-232. [PubMed: 15021209]

25. Byard RW, Krous HF. Sudden infant death syndrome: overview and update. Pediatr Dev Pathol 2003;6:112-127. [PubMed: 12532258]

26. Ponsonby AL, Dwyer T, Cochrane J. Population trends in sudden infant death syndrome. Semin Perinatol 2002;26:296-305. [PubMed: 12211620]

27. Rognum, TO.; Byard, RW. Sudden infant death syndrome, etiology and epidemiology. In: PayneJames, J.; Byard, RW.; Corey, TS.; Henderson, C., editors. Encyclopedia of Forensic and Legal Medicine. Boston: Elsevier; 2005. p. 117-129.

28. Plant LD, Bowers PN, Liu Q, Morgan T, Zhang T, State MW, et al. A common cardiac sodium channel variant associated with sudden infant death in African Americans, SCN5A S1103Y. J Clin Invest 2006;116:430-435. [PubMed: 16453024] 
29. Wang DW, Desai RR, Crotti L, Arnestad M, Insolia R, Pedrazzini M, et al. Cardiac sodium channel dysfunction in sudden infant death syndrome. Circulation. 2006

30. Hong K, Piper DR, Diaz-Valdecantos A, Brugada J, Oliva A, Burashnikov E, et al. De novo KCNQ1 mutation responsible for atrial fibrillation and short QT syndrome in utero. Cardiovasc Res 2005;68:433-440. [PubMed: 16109388]

31. Chen YH, Xu SJ, Bendahhou S, Wang XL, Wang Y, Xu WY, et al. KCNQ1 gain-of-function mutation in familial atrial fibrillation. Science 2003;299:251-254. [PubMed: 12522251]

32. Stengl M, Volders PG, Thomsen MB, Spatjens RL, Sipido KR, Vos MA. Accumulation of slowly activating delayed rectifier potassium current $\left(\mathrm{I}_{\mathrm{Ks}}\right)$ in canine ventricular myocytes. J Physiol (London) 2003;551:777-786. [PubMed: 12819301]

33. Extramiana F, Antzelevitch C. Amplified transmural dispersion of repolarization as the basis for arrhythmogenesis in a canine ventricular-wedge model of short-QT syndrome. Circulation 2004;110:3661-3666. [PubMed: 15569843] 


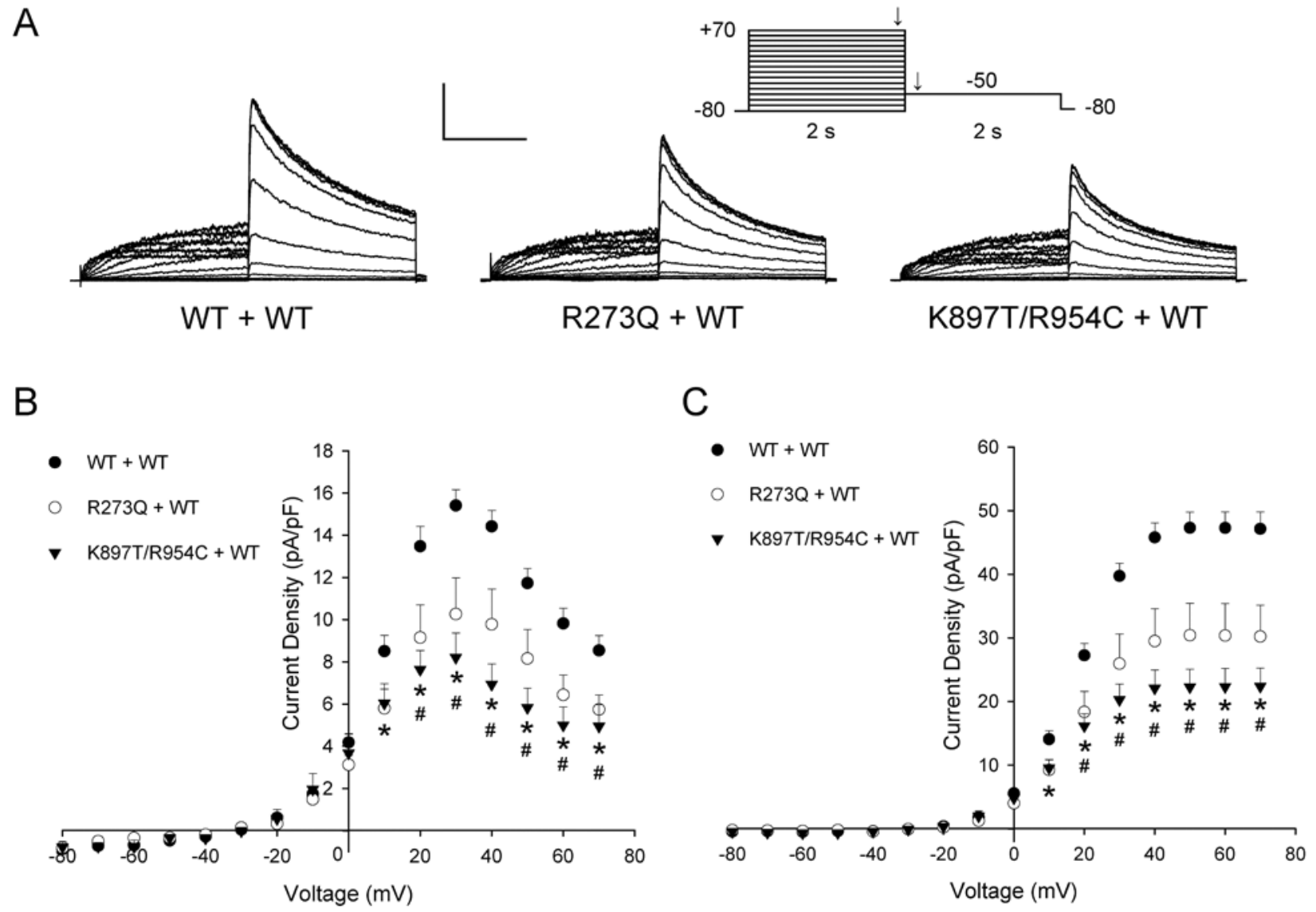

Fig. 1.

Functional characterization of $K C N H 2$ variants R273Q and K897T/R954C. (A) Representative whole-cell current traces recorded from $\mathrm{CHO}$ cells transiently transfected with plasmids encoding WT-HERG, R273Q, and K897T/R954C expressed in tandem with either EGFP or DsRed. In each experiment, the allele listed first in the inset legend is coupled to EGFP while the second is coupled to DsRed. Horizontal and vertical scale bars represent $1000 \mathrm{~ms}$ and 250 $\mathrm{pA}$, respectively. The voltage protocol employed is shown in the inset (arrows indicate the time points at which currents were compared). (B) Current-voltage relationship for potassium current densities (normalized to membrane capacitance) measured in CHO cells expressing WT-HERG + WT-HERG (closed circles, $N=19$ ), R273Q + WT-HERG (open circles, $N=8$ ), or K897T/R954C + WT-HERG (closed triangles, $N=7$ ). (C) Current-voltage relationship for amplitude of peak tail current densities following repolarization to $-50 \mathrm{mV}$ for WT-HERG + WT-HERG (closed circles, $N=19$ ), R273Q + WT-HERG (open circles, $N=8$ ), or K897T/ R954C + WT-HERG (closed triangles, $N=7$ ). Data shown are mean \pm SEM. Significant differences between R273Q + WT-HERG and WT + WT-HERG at the $P<0.05$ level are indicated by *. Significant differences between K897T/R954C + WT-HERG and WT + WTHERG $(P<0.05)$ are indicated by \#. 

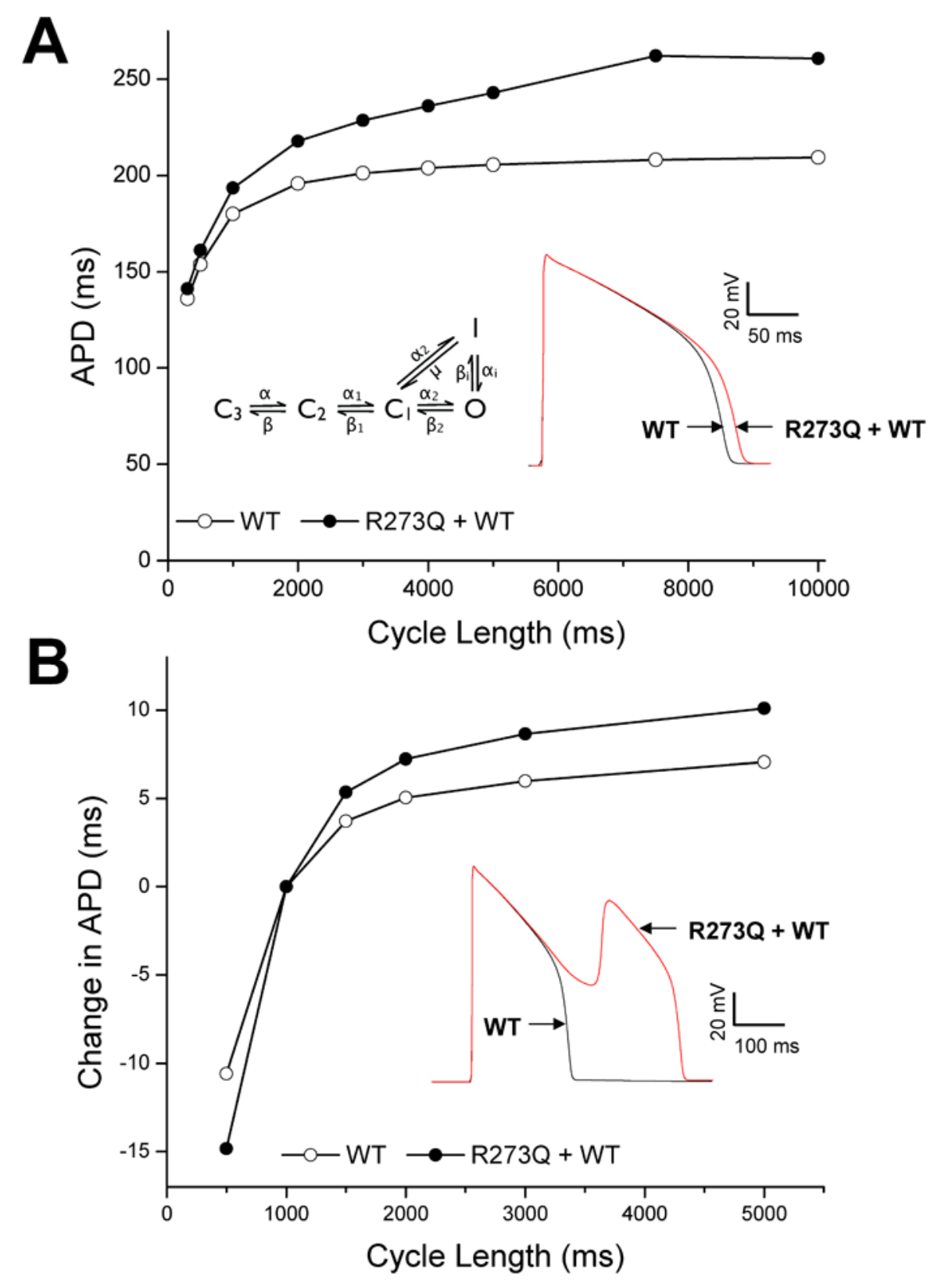

Fig. 2.

Action potential modeling of HERG-R273Q. (A) Predicted APD at various cycle lengths predicted from LRd modeling. The mutant was modeled using data from co-expression with WT-HERG. Inset figures illustrate the Markov scheme used to simulate $\mathrm{I}_{\mathrm{Kr}}$, and a representative overlay of action potentials generated at cycle length $1000 \mathrm{~ms}$. (B) Pausedependent lengthening of APD is illustrated by the relative change in APD at various pause durations. In these experiments, the pause occurred after 50 simulated action potentials when the model was at steady-state. The inset illustrates an EAD occurring following a pause of 7.5 $\mathrm{s}$ for the heterozygous mutant. 

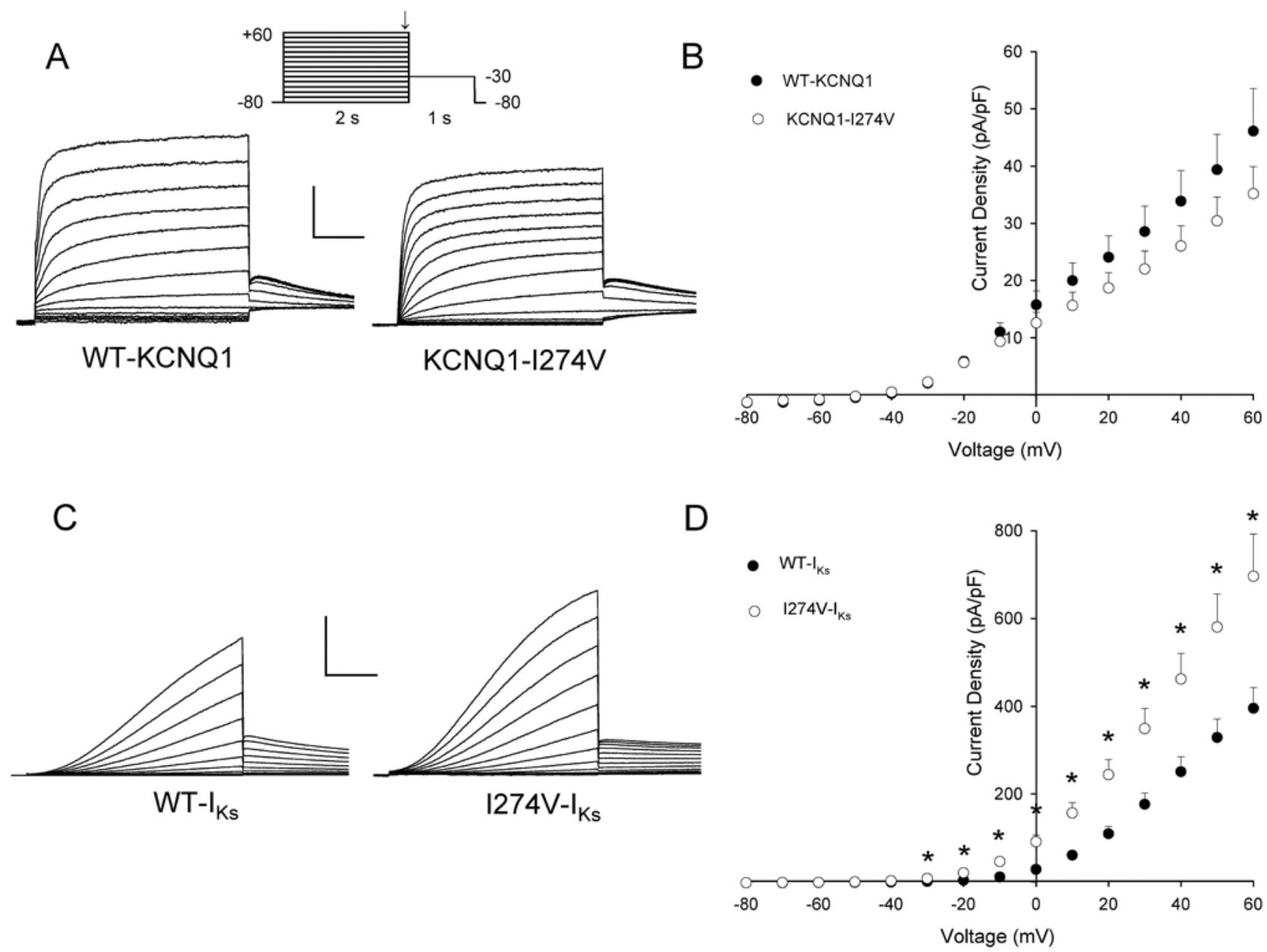

Fig. 3.

Functional characterization of KCNQ1-I274V. (A) Representative whole-cell current traces recorded from CHO cells transiently transfected with WT-KCNQ1 or KCNQ1-I274V. The voltage protocol employed is shown in the inset (arrow indicates the time point at which currents were compared). Horizontal and vertical scale bars represent $500 \mathrm{~ms}$ and $250 \mathrm{pA}$, respectively. (B) Current-voltage relationship for potassium current densities (normalized to membrane capacitance) measured in $\mathrm{CHO}$ cells expressing WT-KCNQ1 (closed circles, $\mathrm{N}=$ 9) and KCNQ1-I274V (open circles, $\mathrm{N}=10$ ). (C) Representative traces illustrating potassium currents observed in CHO cells transiently transfected with WT KCNQ1 + KCNE1 (WT-

$\mathrm{I}_{\mathrm{Ks}}$ ) or KCNQ1-I274V + KCNE1 (I274V-I $\left.\mathrm{I}_{\mathrm{Ks}}\right)$. Horizontal and vertical scale bars represent 500 ms and $2000 \mathrm{pA}$, respectively. (D) Current-voltage relationship for potassium current densities (normalized to membrane capacitance) measured in $\mathrm{CHO}$ cells expressing WT- $\mathrm{I}_{\mathrm{Ks}}$ (closed circles, $\mathrm{N}=9$ ) and $\mathrm{I} 274 \mathrm{~V}-\mathrm{I}_{\mathrm{Ks}}$ (open circles, $\mathrm{N}=10$ ). Data shown are mean $\pm \mathrm{SEM}$. Significant differences between $\mathrm{WT}-\mathrm{I}_{\mathrm{Ks}}$ and $\mathrm{I} 274 \mathrm{~V}-\mathrm{I}_{\mathrm{Ks}}(P<0.05)$ are indicated by $*$. 
A

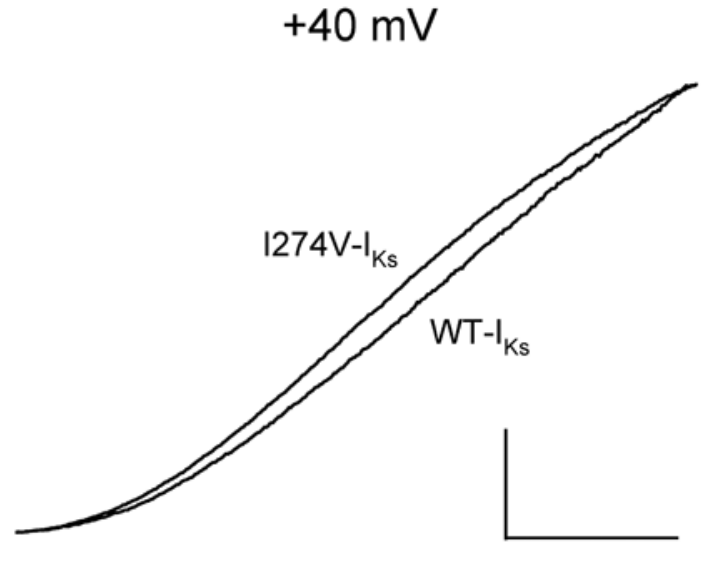

B

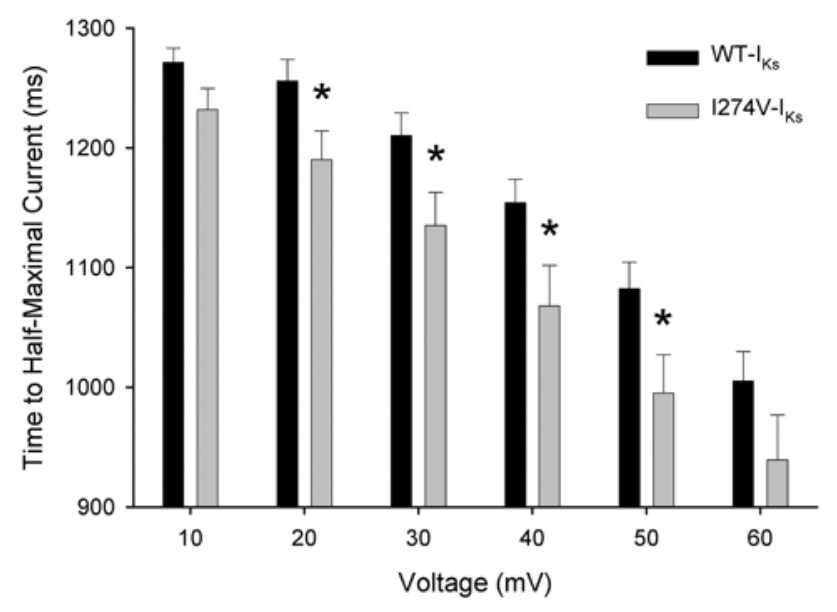

Fig. 4.

Activation kinetics of $\mathrm{I} 274 \mathrm{~V}-\mathrm{I}_{\mathrm{Ks}}$. (A) Representative current traces normalized to peak current illustrating activation of $\mathrm{WT}-\mathrm{I}_{\mathrm{Ks}}$ and $\mathrm{I} 274 \mathrm{~V}-\mathrm{I}_{\mathrm{Ks}}$ during a 2 second pulse to $40 \mathrm{mV}$. Horizontal and vertical scale bars represent $500 \mathrm{~ms}$ and 0.25 arbitrary units (normalized current),

respectively. (B) Voltage dependence of activation. The rate of activation was determined by measuring the time to half-maximal current at each voltage for WT-I $\mathrm{I}_{\mathrm{Ks}}$ (black bars, $\mathrm{N}=9$ ) and I274V-I $\mathrm{K}_{\mathrm{S}}$ (grey bars, $\mathrm{N}=10$ ) from experiments shown in Fig. 2C. Data shown are mean \pm SEM. Significant differences between $\mathrm{WT}-\mathrm{I}_{\mathrm{Ks}}$ and $\mathrm{I} 274 \mathrm{~V}-\mathrm{I}_{\mathrm{Ks}}(P<0.05)$ are indicated by $*$. 


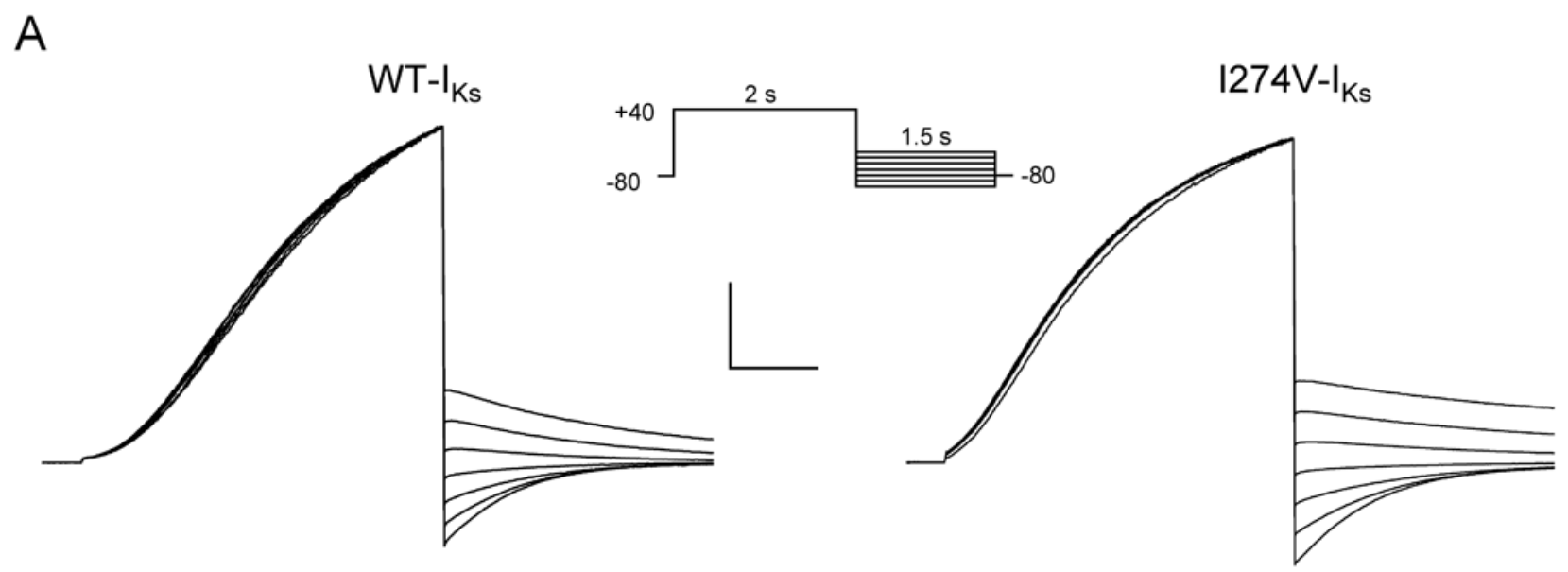

B

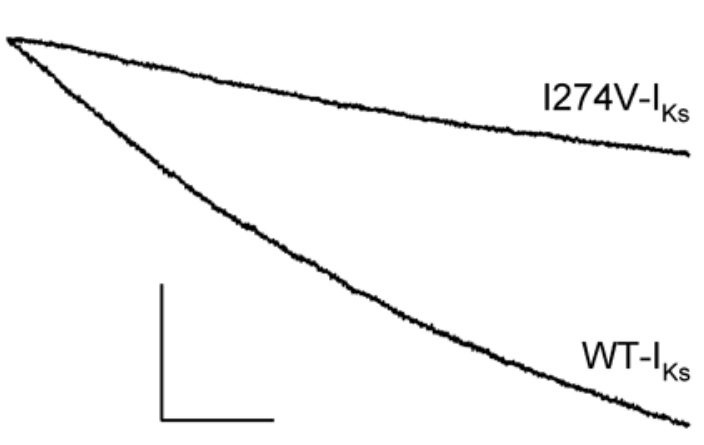

C

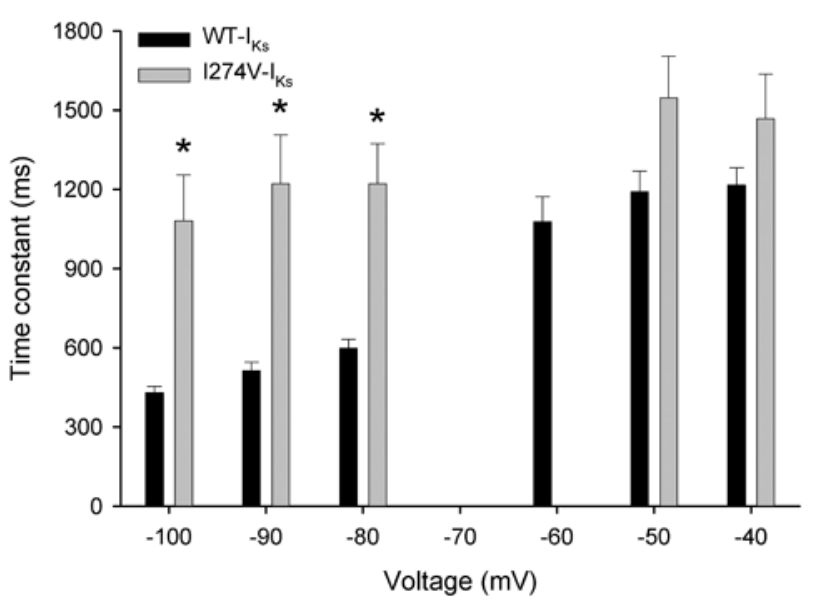

Fig. 5.

Deactivation kinetics of I274V-I $I_{\mathrm{Ks}}$. (A) Representative traces illustrating deactivation in $\mathrm{CHO}$ cells expressing WT- $\mathrm{I}_{\mathrm{Ks}}$ or $\mathrm{I} 274 \mathrm{~V}-\mathrm{I}_{\mathrm{Ks}}$. Currents were evoked by depolarization to $40 \mathrm{mV}$ followed by repolarization to varying negative potentials for $1.5 \mathrm{~s}$. Horizontal and vertical scale bars represent $500 \mathrm{~ms}$ and 0.25 arbitrary units (normalized current), respectively. (B) Averaged, normalized tail current traces illustrating deactivation of WT-I $\mathrm{I}_{\mathrm{Ks}}$ and $\mathrm{I} 274 \mathrm{~V}-\mathrm{I}_{\mathrm{Ks}}$. Deactivation was examined after a $2 \mathrm{~s}$ pulse to $40 \mathrm{mV}$ followed by repolarization to $-40 \mathrm{mV}$. Tail currents were normalized to the peak tail current and are the average of 7 cells. Horizontal and vertical scale bars represent $250 \mathrm{~ms}$ and 0.2 arbitrary units (normalized current), respectively. (C) Voltage dependence of deactivation. Time constants of deactivation were obtained after fitting the tail current with a single exponential equation for $\mathrm{WT}^{-\mathrm{I}_{\mathrm{Ks}}}$ (black bars, $\mathrm{N}=8$ ) or I274V$\mathrm{I}_{\mathrm{Ks}}$ (grey bars, $\mathrm{N}=6$ ). Data shown are mean \pm SEM. Significant differences between WT$\mathrm{I}_{\mathrm{Ks}}$ and $\mathrm{I} 274 \mathrm{~V}-\mathrm{I}_{\mathrm{Ks}}(\mathrm{P}<0.05)$ are indicated by *. 


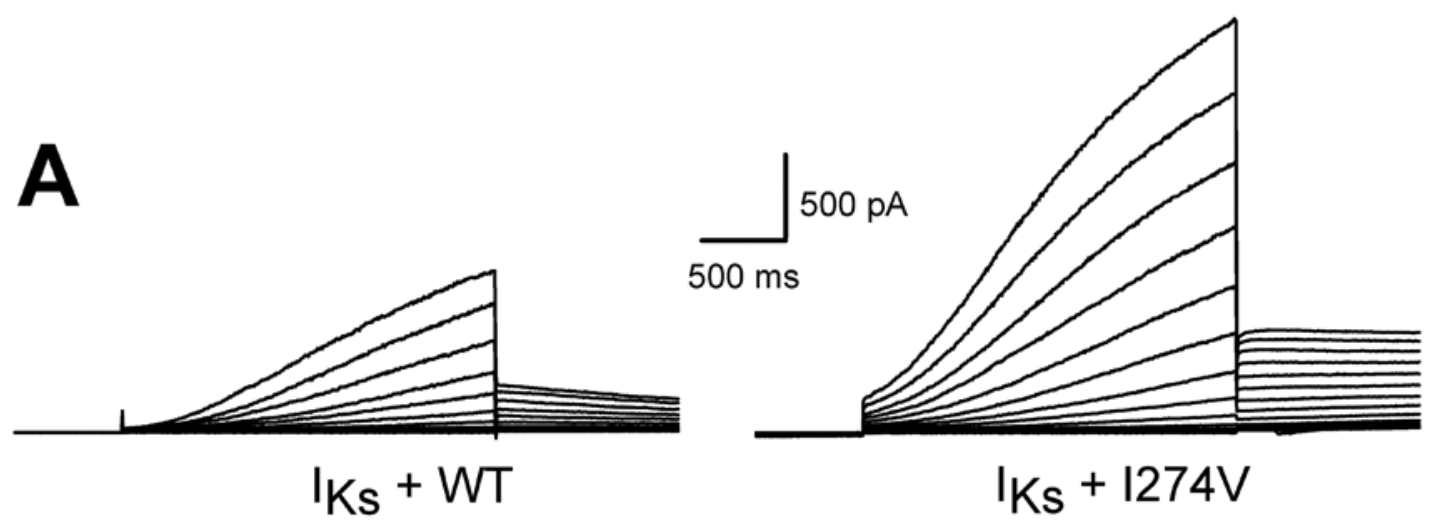

B

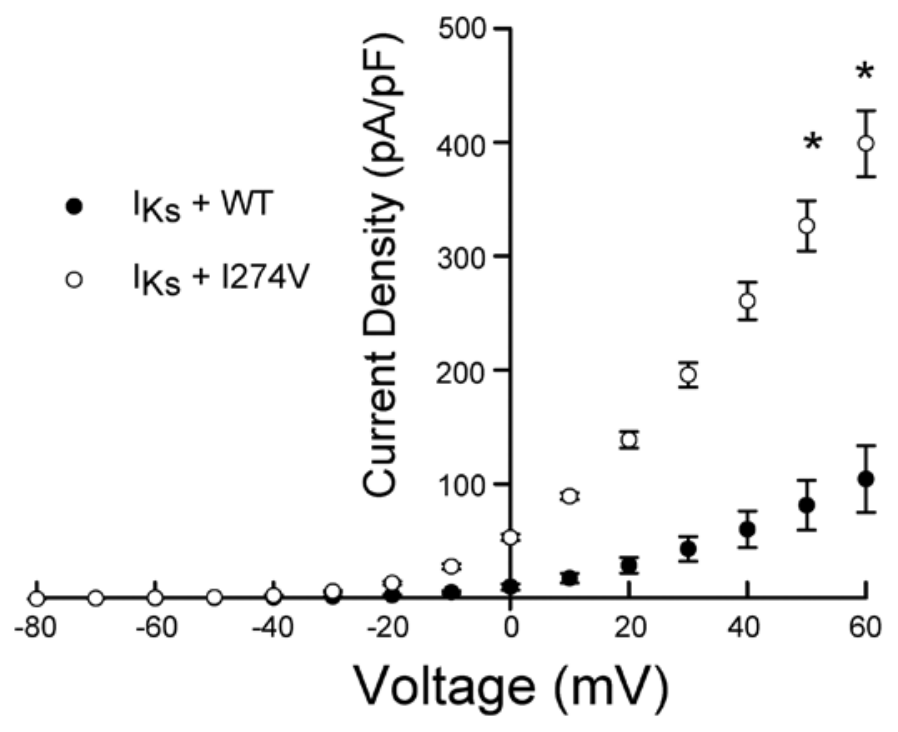

Fig. 6.

Properties of KCNQ1-I274V in the heterozygous state. (A) Representative whole-cell currents recorded from $\mathrm{I}_{\mathrm{Ks}}$ cells transiently transfected with either WT-KCNQ1 or I274V. The voltage protocol used was the same as illustrated in Fig. 3A. (B) Current-voltage relationship for potassium current densities (normalized to membrane capacitance) measured in $\mathrm{I}_{\mathrm{Ks}}$ cells expressing either WT-KCNQ1 (closed circles, $\mathrm{N}=15$ ) or KCNQ1-I274V (open circles, $\mathrm{N}=$ 13). Current density values are different at the $P<0.01$ level for all voltages between -20 and $60 \mathrm{mV}$. 
A

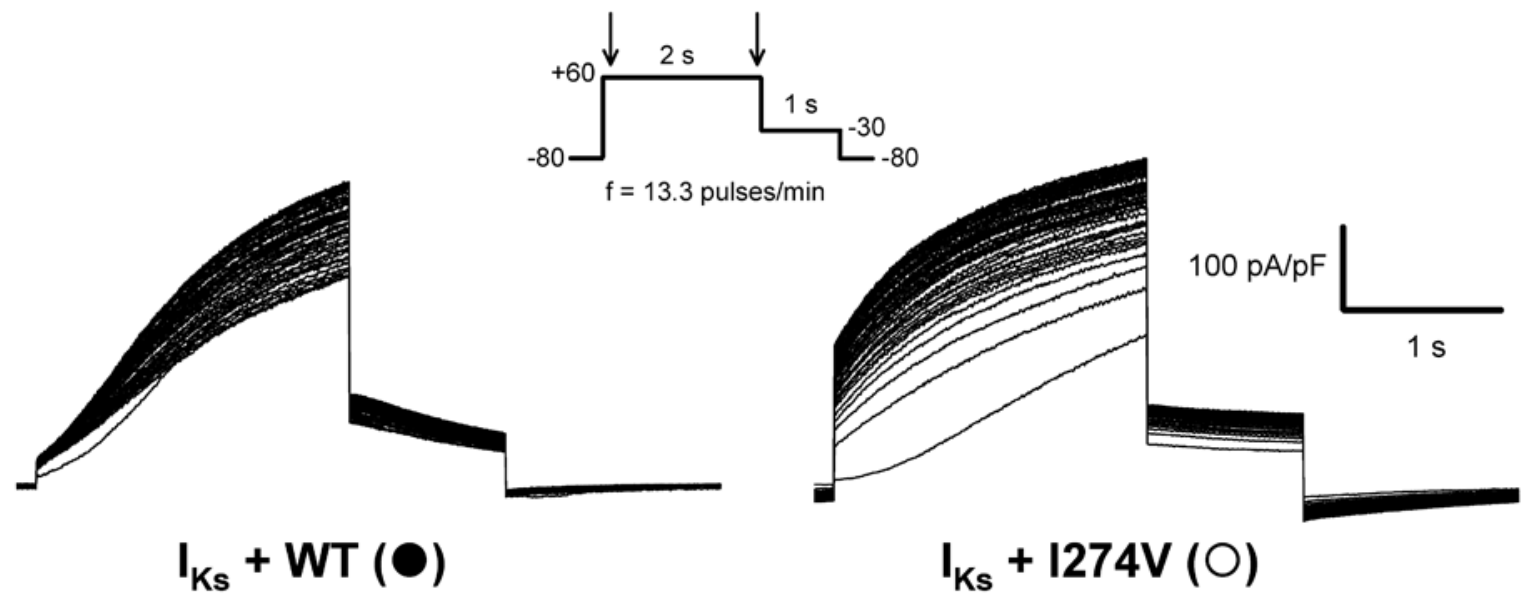

B
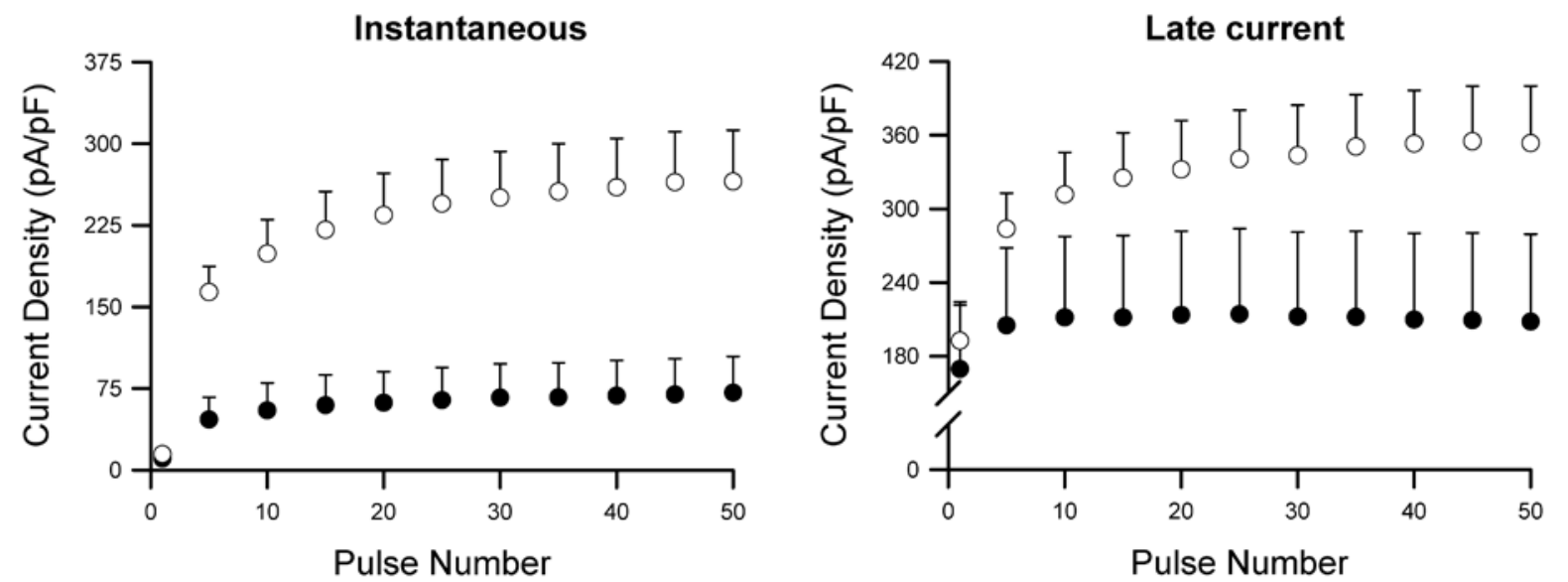

Fig. 7.

Use dependent behavior of $\mathrm{I} 274 \mathrm{~V}$ in $\mathrm{I}_{\mathrm{Ks}}$ cells. (A) Averaged whole-cell current traces recorded from $\mathrm{I}_{\mathrm{Ks}}$ cells transfected with either WT $(\mathrm{N}=8)$ or $\mathrm{I} 274 \mathrm{~V}(\mathrm{~N}=6)$ during a series of 50 depolarizing pulses $(2 \mathrm{~s}, 60 \mathrm{mV})$ delivered at 13.3 pulses/min. Traces shown correspond to pulse numbers $1-10$, and every $5^{\text {th }}$ pulse thereafter. The voltage protocol employed is shown in the inset (arrow indicates the time point at which 'instantaneous' and 'late' currents were compared. (B) Instantaneous current density measured $150 \mathrm{~ms}$ after the start of each depolarizing pulse. Data shown are mean \pm SEM. (C) Late current density measured $1990 \mathrm{~ms}$ after the start of each depolarizing pulse. Data shown are mean \pm SEM. 
A

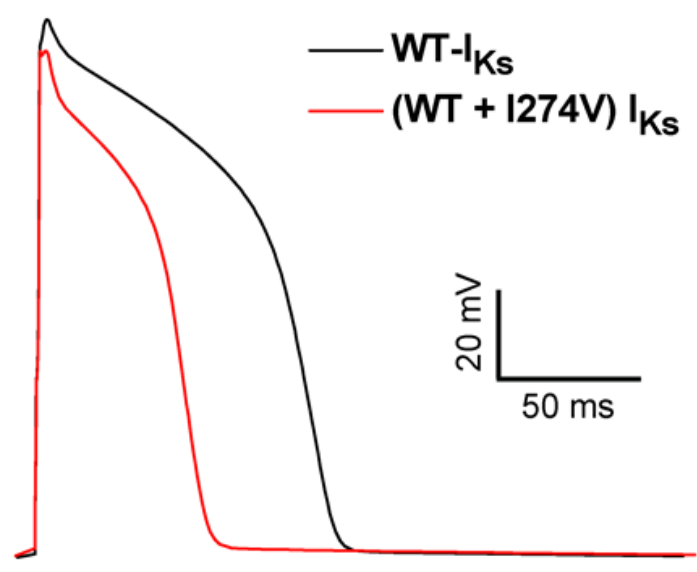

$300 \mathrm{~ms}$ cycle length

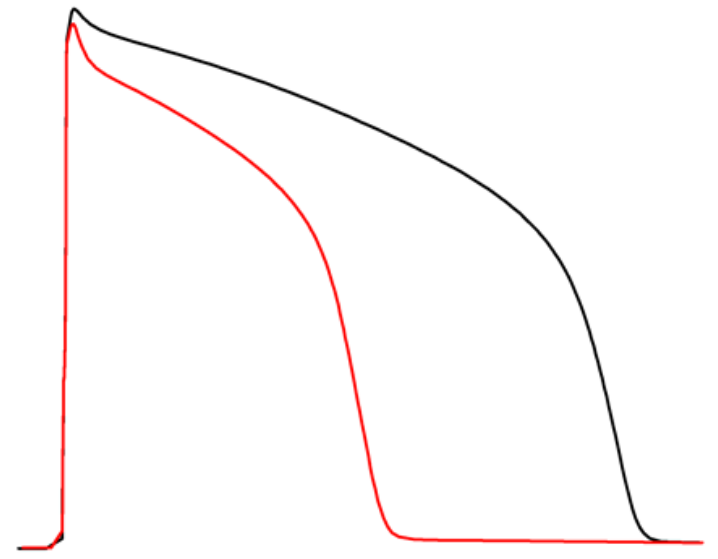

1000 ms cycle length
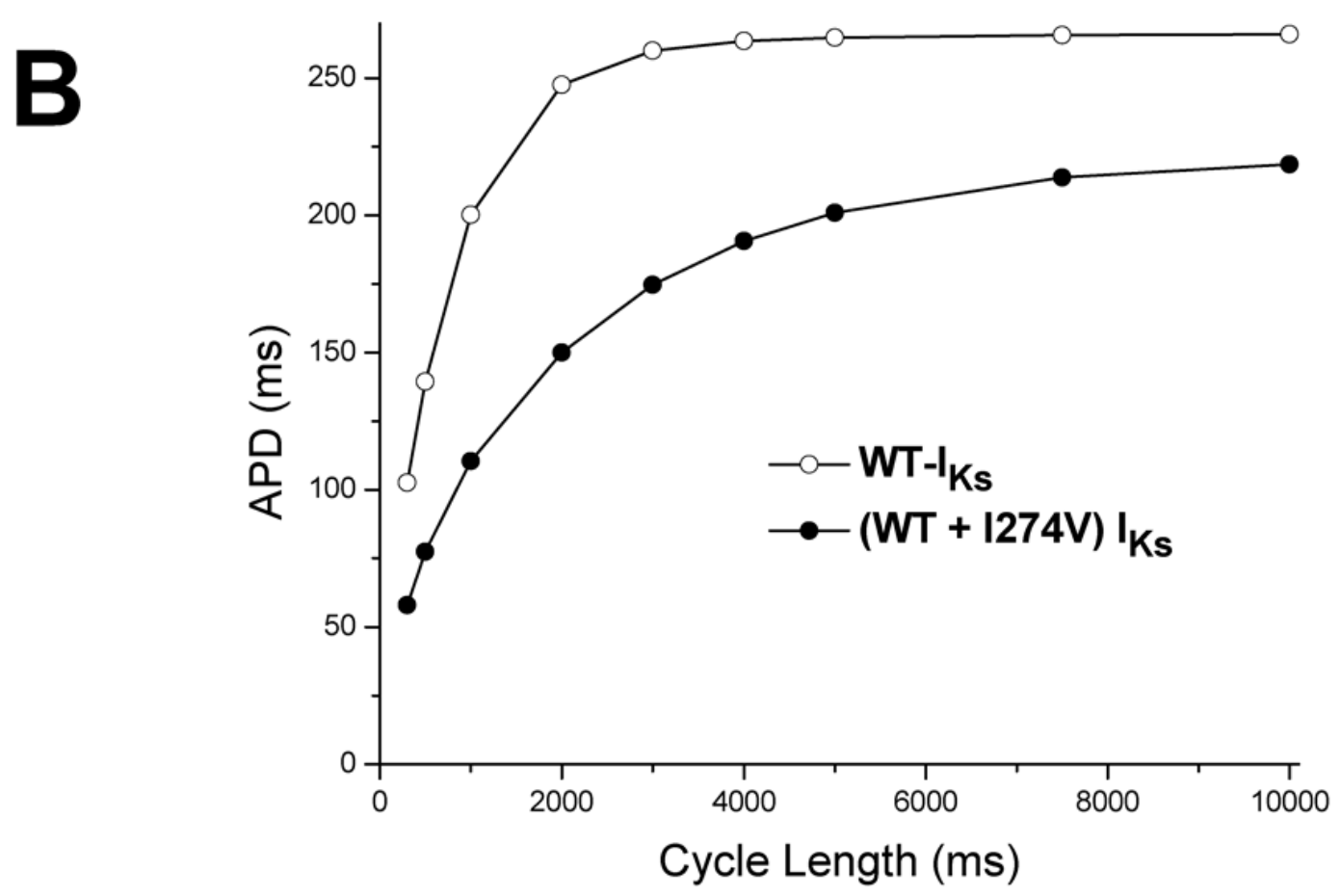

Fig. 8.

Action potential simulations with $\mathrm{WT}-\mathrm{I}_{\mathrm{Ks}}$ and $\mathrm{I} 274 \mathrm{~V}-\mathrm{I}_{\mathrm{Ks}}$ (A) Simulated action potentials using WT- $\mathrm{I}_{\mathrm{Ks}}$ (black line) and heterozygous $\mathrm{I} 274 \mathrm{~V}-\mathrm{I}_{\mathrm{Ks}}$ (red line) at cycle lengths of $300 \mathrm{~ms}$ and 1000 ms. Data for these simulations were derived from the expression experiments in $\mathrm{I}_{\mathrm{Ks}}$ cells (Fig. 6 and Fig. 7) and the modeled currents are illustrated in supplemental Fig. S3. (B) Relationship of APD with cycle length for WT- $\mathrm{I}_{\mathrm{Ks}}$ and heterozygous $\mathrm{I} 274 \mathrm{~V}-\mathrm{I}_{\mathrm{Ks}}$. 